\title{
Quality Factor Improvement for Nano Cavity
}

\author{
Mohammed Nadhim Abbas \\ Electrical Engineering Department, \\ College of Engineering, \\ University of Baghdad, Baghdad, Iraq
}

\author{
Duaa S. Mohammed \\ Electrical Engineering Department, \\ College of Engineering, \\ University of Baghdad, Baghdad, Iraq
}

\begin{abstract}
In this paper, the increasing of quality factor in the stimulated amplification of surface plasmon polaritons at visible frequencies was reported. Laser science has been successful in producing high powered, faster, and smaller coherent light sources. Such lasers are restricted, both in optical mode size and physical device dimension, to being larger than half the wavelength of the optical field, and it remains a key fundamental challenge to realize ultra-compact lasers that can directly generate coherent optical fields at the nanometer scale, far beyond the diffraction limit. A way of addressing this issue is to make use of surface plasmons, which are capable of tightly localizing light, but so far ohmic losses at optical frequencies have inhibited the realization of truly nanometer-scale lasers based on such approaches. We design a plasmonic laser with quality factor equal 900 at wavelength $500 \mathrm{~nm}$.
\end{abstract}

\section{General Terms}

Laser, Plasmonic Laser, nanoparticles.

\section{Keywords}

Plasmonics, Surface plasmon polration, Localized surface plasmon resonance, nanocavity,

\section{INTRODUCTION}

Plasmon lasers are a new type of cohesive optical amplifiers that generate and support light well below its diffraction Limit. Their intense, and confined optical fields can improve significantly light-matter interactions and bring fundamentally new capabilities to bio-sensing, data storage, photolithography and optical communications [1]. One of the most common problem of integrated photonic is diffraction limit, this problem can be solved by using a metallic Nano cavity. But the metallic cavity has high ohmic loss $[1,2$, and 3 ] which led to low quality factor and limited laser work at room temperature. The sufficient gain was adopted to mitigate losses, while using hybrid semiconductor insulator-metal nanostructure for strong confinement with low metal loss. High cavity quality factors, approaching 650 due to using spp technique, along with strong mode confinement, lead to enhancements of spontaneous emission rate. By controlling the structural geometry the cavity modes could be reduced to achieve single- and two-mode lasing. Plasmonic lasers realize optical confinement and feedback using surface plasmon polaritons (SPPs), quasiparticles of photons and electrons at metal/dielectric interfaces, which can be amplified by suitable optical gain media. The high gain of crystalline semiconductors is typically necessary to overcome fast electron scattering in metals (10 fs), which leaves plasmonic lasers with high parasitic cavity loss. Nevertheless, SPPs offer the capability to reduce optical mode sizes far below the scale of the vacuum wavelength, leading to compact lasers that can generate extremely focused optical excitations on potentially ultrafast timescales [4].
Plasmonic laser with multiple configuration can confined light below its diffraction limit because of different relative permttiviies, $\varepsilon$, of metal and the surrounding dielectric material [4]. The concentration of light below the diffraction limit using metal lead to an electric field enhancement [4], laser threshold [5] and heat sink [7]. With respect to classical lasers, Plasmon lasers amplify light coupled to oscillating electrons, low power and higher speed [6]. Plasmon laser adds momentum to light enabling their physical size and mode volume to shrink below the diffraction limit [6]. Having an ultra-high speed -low power will open new possibilities for optical signal processing making it with electronics in high performance region $[6,7]$. However, using metal to reduce size of the laser and provide heat sink, it has losses, higher than that at dielectric cavities, the energy dissipated due to collision electrons so SPPs can travel only short distances. This leads to the small value of a lifetime of free electrons and then few store energy and few quality factors which represent an important factor in cavities. But using low index dielectric layer can enhance quality factor by reducing losses due to using metal [8]. Although there is many challenges which discussed the construction of nano-laser is similar to that of conventional laser. SP generated at metal interface and amplify by a dielectric structure which represent a gain medium, the gain medium amplifies the SP by stimulated emission, while feedback mechanism allow SP to resonate. By varying the frequencies of the incident light, different surface charge density distributions can be generated for long-range SPPs propagation. The important part in the laser is its cavity which we will improve its quality factor. Several plasmonic laser reported using sandwich layer to enhance quality factor. Room temperature, high q-channel waveguide surface plasmon Nano cavity suggest using different width waveguide to enhance the quality factor [8], but its quality factor equal 300 at room temperature

\section{PLASMONIC LASER}

\subsection{Study Model}

Here, three dimensional plasmonic laser was reported (with cylindrical cavity) optical confinement. The cylindrical cavity with a radius of $100 \mathrm{~nm}$ and length of $1 \mathrm{um}$ which consist of indium phosphate (InP), air, $\mathrm{SiO} 2$ and silver which confine light in the cavity. Model geometry is depicted in figure (1). The InP layer (with refractive index 3.4 [9]) thickness is $100 \mathrm{~nm}$ which separated from the silver surface by using a layer of silica, where InP represent the active material in this laser cavity. The $\mathrm{SiO} 2$ is a low refractive index layer with refractive index 1.5 [9]. A rectangular structure of silver was surrounded a cylinder of InP and rectangular of air. The reason of using circular form is to get higher confinement and reduce losses $[1,14]$, where the nanowire laser on the surface of metal substrate reduces metal loss, but had limited feedback which requires cavity with length higher than the wavelength $[1,15]$. To mitigate limited feedback we made the 
cavity length more than wave length. And the plasmon losses can be compensated by using InP gain material [8]. The thickness of the cavity is changed to control quality factor value and confinement factor. Total height of the structure is $(500 \mathrm{~nm})$ in the $\mathrm{z}$-direction and total width $(500 \mathrm{~nm})$ in the $\mathrm{y}$ direction, which contain from silver (table for different values of silver refractive indices is from ref. [9]. Numerical simulation using COMSOL multiphysics is conducted to the model shown in figure (1). A plane of the TM polarized electromagnetic field is incident from the top of the cavity in the ear region. Due to principle of surface plasmon polaration (SPP) the wave will propagate in the dielectric layer $(\mathrm{SiO} 2$ layer). Using of the dielectric layer is to reduce the absorption by metal [10], and to compensate losses of metal at the resonance with the total internal reflection due to the interface SPPs provides an amplification channel via the external gain medium that avoids the fluorescence quenching effect inside the Nano slit [11].

(a)

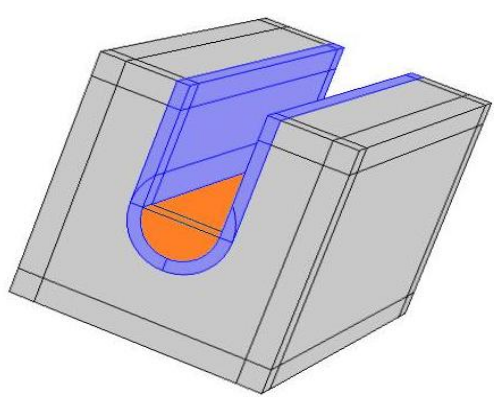

(b)

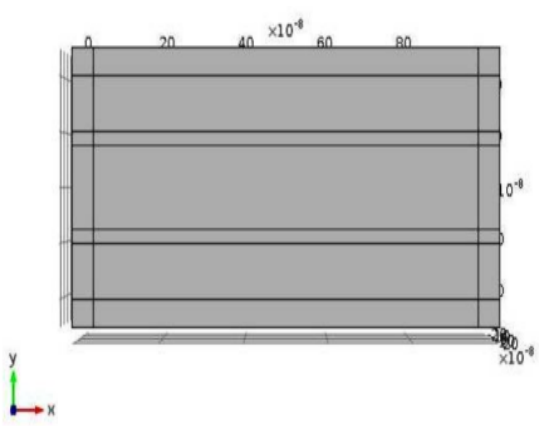

(c)

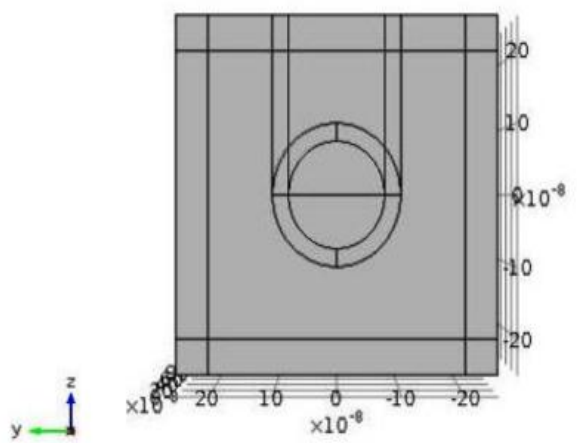

Figure(1) geomatry of metallic cavity (a) 3D view of the geomatry each matrial is shown in different color sio2 layer in blue, inp layer in orange and silver in gray color. (b) top view (x-y view) of the geomatry. (c) front view $(y-z$ view)of geomatry shown the circular shap of the cavity.

\subsection{Quality Factor of Cavity}

Quality factor of cavity can be defined as a ratio of energy stored in cavity to energy dissipated per oscillation cycle. Q factors of SPP cavities always smaller than those of conventional cavities [8].There is many parameters affect on quality factor of the cavity (Q.F), one of these parameters is high losses due to using metal in plasmonic cavity $[5,6,7$, 8,12 and 13].There are many models suggested to increase Q.F. for plasmonic cavities by using hybrid cavity [12], different width cavity [8], using array of nano-cavity [13] and nano-wire cavity [5]. All previous solutions depend of using dielectric layer to reduce losses of the metal and then increase quality factor of the laser, because quality factor value inversely proportional to losses, as show in equation 1.1

$$
\mathrm{Q}=2 \pi \nu \ell / \mathrm{c} \gamma
$$

Where $v$ is the frequency of light, $\ell$ is the distance between cavity mirrors, $\mathrm{c}$ is a speed of light and $\gamma$ is the cavity loss. From equation (1.1). Quality factor could be increased either by reduce losses or by increasing a distance between mirrors of the cavity. In this work, use low index material in metal semiconductor interface and cylindrical cavity was suggested to get high quality factor. The low index layer allows the electric fields of the cavity mode inside metal to decrease, while maintaining good confinment[8].

\section{RESULT AND DISCUSSION}

The electric field norm $(\mathrm{V} / \mathrm{m})$ and current density norm $\left(\mathrm{A} / \mathrm{m}^{2}\right)$ are calculated along the metal-dielectric interface (as shown in figure 2). From the figure we noticed that most of the energy concentrate at the dielectric layer, and the SPP waveguide mode is strongly confined in the bottom of the cavity $(\mathrm{Ag} / \mathrm{InP}$ interface). The introduction to low index dielectric layer increase quality factor by decrease losses in the metal which contains due to penetration depth, because this dielectric layer reduce the absorption of metal [11].the absorption in the metal is the main to compensate the metal loss to increase the quality factor. The losses (see figure 2, d) are concentrated in the region between metal and dielectric, one of the reasons for this loss is the penetration depth. This losses can be compensated by using gain material InP [14]. When the wave is resonate between mirrors of laser will attenuated due to high losses of metal mirrors, but when pass through the gain material compensate loss

(a)

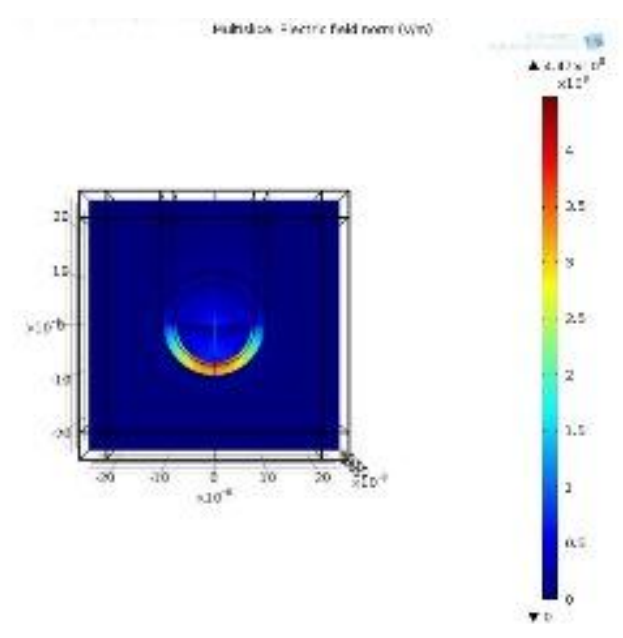


(b)

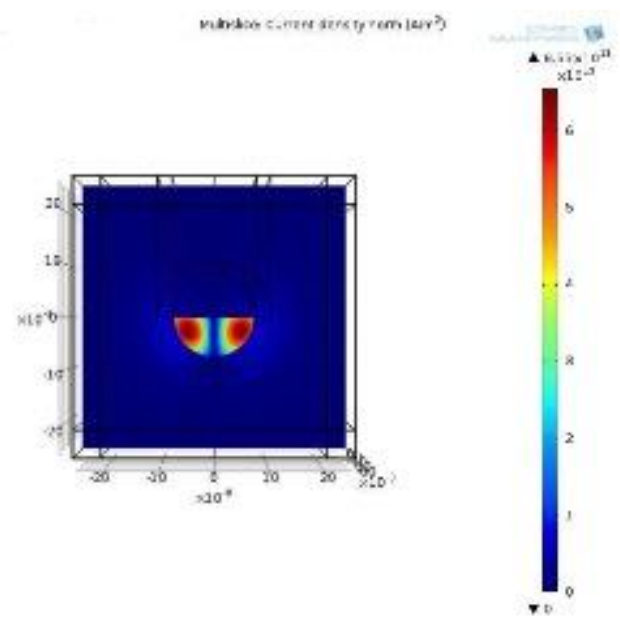

(c)

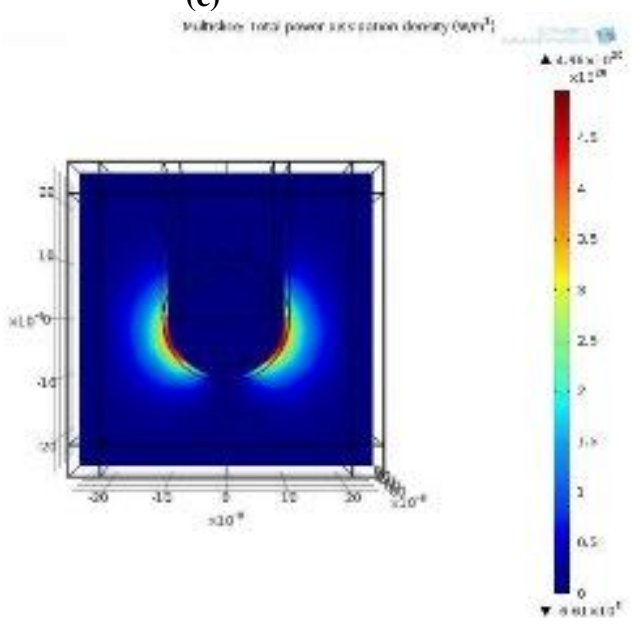

Figure (2): Energy distribution in the structure. a) Electric field norm (V/m). b) Current density. c) Losses in the structure.

The using of metal is confined light in 3D [8] (see figure 2, a). When thickness of dielectric layer increases (absorption of metal decrease) the value of quality factor increases too (as shown in figure 3,4). Figure (3) shows different values of quality factor calculation versus wavelength for different width of dielectric layer width. We get quality factor reach to 900. But the increasing of quality factor due to increase of low index material limited by confinement factor. The confinement factor decrease with increase thickness of dielectric layer.

From this experiment noticed that, there is other factors effect on quality factor like radius of cylindrical cavity, length of the cavity, the ratio of the dielectric layer thickness to active material thickness, and type of low index material used in the cavity. Figure (3) show the relation between increasing of quality factor with increasing of dielectric layer thickness. The change thickness of dielectric layer shifted the resonance wave length but this shift is very small to be notice so that in out experiment neglected this shift.

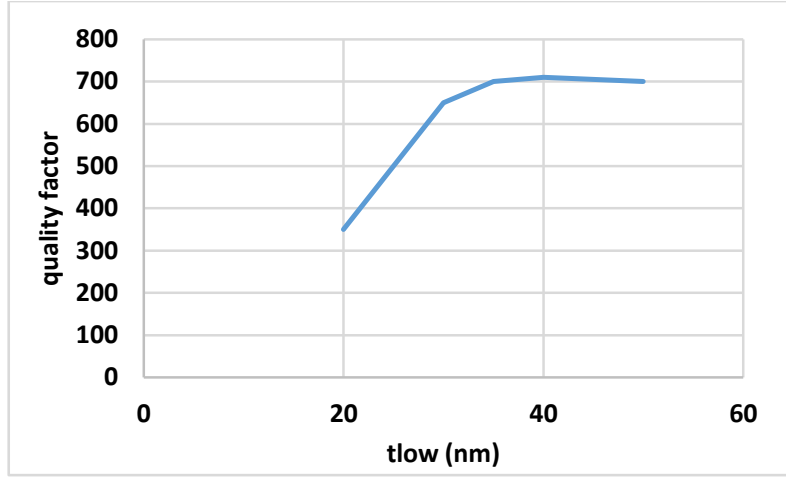

Figure (3): relation between increasing of quality factor values with increasing of dielectric layer thickness.

From figure (3) noticed that the quality factor values saturated when thickness reach $40 \mathrm{~nm}$ and above. This because using the radius of cavity $100 \mathrm{~nm}$ only, if the cavity radius increased this saturated value will shifted.

Figure (4) show shifted resonance wave length with increase cavity radius because the distance between mirrors depend on wavelength of light wave.

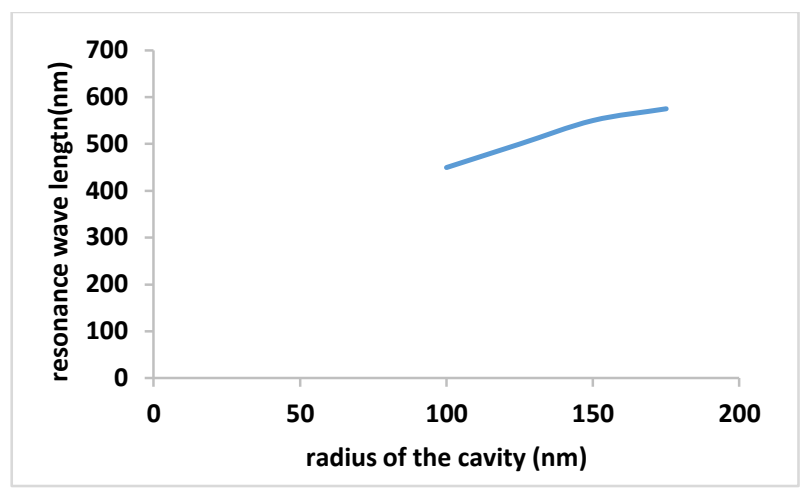

Figure (4): resonance wave length versus radius of the cavity.

\section{CONCLUSION}

A room temperature plasmonic laser had been demonstrated with strong mode confinement much smaller than diffraction limit high quality factor plasmonic laser. The electric field resonance of the plasmonic Nano-laser was investigated, considering impact of changing the length, width of laser and thickness of dielectric layer. From simulation we notice that using of dielectric layer improve quality factor. The quality factor was enhancing from 300 to be 900 when using dielectric layer compared with model without dielectric layer while small mode volume are maintained. Moreover the laser threshold was decreased It is also notice that the dissipated power due to losses of the using metal reduced. Next work, try using an array of cavities to enhance the quality factor by coupling technique.

\section{REFERENCES}

[1] Ren-Min Ma, Rupert F. Oulton, Volker J. Sorger, Guy Bartal1 and Xiang Zhang," Room-temperature subdiffraction-limited plasmon laser by total internal reflection", Nature letter (2010)

[2] C. Breck Hitz, J. J. Ewing, Jeff Hecht, "Introduction to Laser Technology", 4th Edition, April 2012, Wiley-IEEE Press. 
[3] Yu-Cheng Hsu, Kuok-Pan Sou, Shih-Pang Chang, KungShu Hsu, M. H. Shih et al, "Room temperature ultraviolet GaN metal-coated nanorod laser", APPLIED PHYSICS LETTERS 103, (2013).

[4] William L. Barnes, Alain Dereux \& Thomas W. Ebbesen, "Surface plasmon subwavelength optics", NATURE (2003).

[5] Stefan A. Maier, "Ultrafast plasmonic nanowire lasers near the surface plasmon frequency", (NATURE PHYSICS, 2014).

[6] Martin T. Hill, "Status and prospects for metallic and plasmonic nano-lasers", J. Opt. Soc. Am 2010

[7] Yu-Jung Lu, Chun-Yuan Wang, Jisun Kim, Hung-Ying Chen, Ming-Yen Lu, Yen-Chun Chen , Wen-Hao Chang, Lih-Juann Chen,Mark I. Stockma,n,Chih-Kang Shih, and Shangjr Gwo, "All-Color Plasmonic Nanolasers with Ultralow Thresholds: Autotuning Mechanism for SingleMode Lasing", nanolatter (2014).

[8] Ju-Hyung Kang,1 Hong-Gyu Park,1 and Soon-Hong Kwon , "Room-temperature high-Q channel-waveguide surface plasmon nanocavity" (optic express ,2011)
[9] E.D. Palik, Handbook of Optical constants of solids (Academic press, 1998).

[10] V. Dolores-Calzadilla, D. Heiss,1 A. Fiore and M. Smit, "Waveguide-Coupled Nanolasers in III-V Membranes on Silicon", ICTON 2013

[11] Jae Woong Yoon, Seok Ho Song and Robert Magnusson, "Ultrahigh-Q metallic nanocavity resonances with externally-amplified intracavity feedback", SCIENTIFIC REPORTS 2014.

[12] Qing Wang, "Hybrid photonic-plasmonic molecule based on Metal/Si disks", (optic express, 2011).

[13] Wei Zhou," Lasing action in strongly coupled plasmonic nanocavity arrays", (NATURE NANOTECHNOLOGY, 2013).

[14] Ren-Min Ma, Xiaobo Yin, Rupert F. Oulton, Volker J. Sorger, and Xiang Zhang, Multiplexed and Electrically Modulated Plasmon Laser Circuit, nanoletter (2012).

[15] Oulton, R. F. et al. Plasmon lasers at deep subwavelength scale. Nature 461, 629_632 (2009). 